\title{
Personal Health Informatics. Editorial
}

\author{
C. A. Kulikowski', A. Geissbuhler ${ }^{2}$ \\ ' Department of Computer Science, Rutgers-The State University of New Jersey, New Brunswick, NJ, USA \\ ${ }^{2}$ Department of Imaging and Medical Informatics, Geneva University Hospitals, Geneva
}

\section{Introducing the 2012 Yearbook and its Theme on Personal Health Informatics}

The Yearbook includes a number of articles focusing on the theme of Personal Health Informatics. It covers recent informatics contributions and points to future directions on how the gradual incorporation of personalization capabilities is enabling more focused and individualized approaches to patient care and the answer of patient-focused clinical and translational research questions. This theme is especially relevant given the high expectations that accompany the push into translational applications of genomic and other -omic data for health care at the level of the individual patient. The dissemination of health informatics methods worldwide is a major goal of IMIA, and personalization issues are at the center of the challenges facing both researchers and clinicians as they strive to bridge the divide between burgeoning -omics research, and the pressures of clinical practice. There are serious ethical and societal challenges that arise from personalization in terms of the requirements for security and confidentiality of individual data that can also impact families and others related to a patient. On the research side, there are issues of data and knowledge integration and interpretation - as well as the management of massive datasets, especially when imaging and whole genome data must be analyzed concurrently.

The Keynote by Enrico Coeira argues persuasively for the need for better ways of envisioning and implementing systems that will truly help personalize the decisions made by patients as well as health care providers. He argues that the field will need to break from its predominantly biological, populationlevel, and generic view of diseases, treat- ments, and their informational content, and begin to take into account the many individual perceptual and cognitive human factors which define the clinical context involved in personal healthcare.

The 2012 IMIA Yearbook includes the following articles:

Keynote on the Theme of Personal Health Informatics - Enrico Coiera

Editorial, IMIA President Statement, Synopses and summaries of Best Paper Selections in each of the subfields of biomedical informatics.

Surveys authored in selected subfields of health informatics include:

- Michael Rigby (PHI/CI): Personal Health, Person-centred Health and Personalised Medicine - Concepts, Consumers, Confusion and Challenges in the Informatics World

- Christophe Gaudet-Blavignac (HCM): Serious games in health care: a survey

- Sabine Koch (HF): Critical advances in bridging personal health informatics and clinical informatics

- Bob Gann (HIS): Giving choice and control: health informatics on the patient journey

- Katarzyna Wax (SSII): Smartphone in My Pocket: From Sensor Informatics to Personal, Pervasive Health Informatics

- Tze-Yun Leong (DS): Toward Patient-Centered, Personalized and Personal Decision Support and Knowledge Management: A Survey

- Nigam Shah: Translational Bioinformatics embraces Big Data

IMIA Working Group Papers:

- Chris Paton: Five Self-Tracking and Self-Experimentation Using Social Media for Patient Empowered Self-Care

- JA Seoane: Data Integration in genomic medicine: trends and applications

- George Demiris: Smart Mobilizing Older Adults: Harnessing the Poten- 
tial of Smart Home Technologies

- Shashi B Gogia: Personal Handheld Computing Devices for Personalizing Healthcare

- Elizabeth Borycki: Empowering Patients: Making Health Information and Systems Safer for Patients and the Public

- Simon de Lusignan: Key concepts to assess the readiness of data for International research: Data quality, lineage and provenance, extraction and processing errors, traceability, and curation

Research \& Education:

- Arie Hasman: IMIA accreditation of Health Informatics programs

- Portia Grace Fernandez-Marcelo: Emerging eHealth Directions in the Philippines

History of Medical Informatics:

- Marius Fieschi: The development of $\mathrm{AI}$ in medicine and the research environment of the SPHINX project at the start of the 1980s

- Valerio Yácubsohn: A Short History of the Beginnings of Hospital Information Systems in Argentina

\section{About the Yearbook of Medical Informatics}

The Yearbook of Medical Informatics of the International Medical Informatics Association (IMIA) is distributed through IMIA's Member and Corresponding Member Societies worldwide. Since its inception in 1992 it has been the single most important publication summarizing the activities of IMIA, and showcasing the best of medical informatics research for the previous year. Its original survey articles give overviews of recent developments. The Yearbook remains a non-profit publication of IMIA, jointly published with Schattauer Verlag. It is currently subscribed to by 23 of IMIA's member societies, providing access for about 25.000 individual members.

We are very pleased to announce that a number of very experienced and committed colleagues have accepted the responsibilities of leading the editorial team of the IMIA Yearbook for 2013. They are Marie-Christine Jaulent (CRC - Centre de Recherche de Cordeliers) and Brigitte Seroussi (Assistance PubliqueHopitaux de Paris), France, and Christoph Lehman (Johns Hopkins), USA. We look forward to their bringing new and exciting ideas for the ongoing development of the IMIA Yearbook, while maintaining its tradition of balanced scientific and practical assessment of the research progress in our field as it evolves in future years.

\section{Information on IMIA and on its Regional Groups}

The Yearbook contains detailed information about IMIA, its Member Societies, Regional Groups, Working Groups, and Special Interest Groups. A detailed report on the activities of IMIA regions is included with the help of Regional Editors. We would like to thank Rolf Engelbrecht (for EFMI), Michio Kimura (for APAMI), Ghislain Kouematchoua (for HELINA), Carol Hullin (for IMIA LAC), Ramin Moghaddam (for MEAHI) and Karen Greenwood and RoseMary MacVicar-Elliot (for the North American IMIA Member Societies) for their valuable contributions.

\section{Acknowledgements}

The editors gratefully acknowledge the contributions of the referees and guest editors. They would also like to thank the authors of the invited surveys, reviews and the historical paper,s and the contributors to the Research and Education Section. They are most appreciative of the considerable skill, time, and effort devoted by Christian Lovis, the Managing Editor, and by the Section Editors, Claudine Bréant, Célia Boyer, Adrien Depeursinge, Arnaud Gaudinat, Alberto Guardia, Daniel Kubias, Rodolphe Meyer, Henning Müller, AnneMarie Rassinoux, Patrick Ruch, and Lina Yip. They especially wish to thank the Editorial Assistant, Martina Hutter, from the Medical Faculty at the University of Heidelberg, without whose untiring efforts the Yearbook would not have been completed. The editors appreciate the contributions of the Advisory Board to the planning of this Yearbook. They are Reinhold Haux, Victor Maojo, Georges Mihalas, and Peter Murray.

The referees who contributed to the selection of articles in the 2012 Yearbook of Medical Informatics were:

Samantha A. Adams, The Netherlands

Kouhei Akazawa, Japan

Elske Ammenwerth, Austria

Manfredo Atzori, Switzerland

Samy A. Azer, Saudi Arabia

Fernan Gonzalez Bernaldo de Quiros, Argentina

Olivier Bodenreider, USA

Alex A.T. Bui, USA

Anita Burgun, France

Jean Charlet, France

Ronald Cornet, The Netherlands

Karen Courtney, Canada

Stefan Darmoni, France

Dina Demner Fushman, USA

John T. Donnelly, USA

Martin Dugas, Germany

Walter Gall, Austria

Dragan Gamberger, Croatia

Natalia Grabar, France

Stefan Gräber, Germany

Thierry Hamon, France

Michael Hogarth, USA

Andreas Holzinger, Austria

Dimitar Hristovski, Slovenia

Josef Ingenerf, Germany

Marie-Christine Jaulent, France

Katharina Kaiser, Austria

Jeongeun Kim, Korea

Farah Magrabi, Australia

Stéphane Meystre, USA

Jeannette Murphy, United Kingdom

Toshiharu Nakai, Japan

Jean Marc Palm, Canada

Philip Payne, USA

Natalia Pletevna, Switzerland

Michael Poprat, Germany

Daniel Racoceanu, Singapore

Rainer Röhrig, Germany

René Schneider, Switzerland

Lauge Soerensen, Denmark

Selma Supek, Croatia

Guy Tsafnat, Australia

Manolis Tsiknakis, Greece

Alexander Turchin, USA

Frank Ückert, Germany

Alfonso Valencia, Spain

Toru Yao, Japan

Xue Zhong Zhou, USA

Tatjana Zrimec, Australia

Pierre Zweigenbaum, France 